\title{
Tabu Search Type Algorithms for the Multiprocessor Scheduling Problem
}

\author{
Olivier BUFFET* $\quad$ Liliana CUCU ${ }^{\dagger}$
}

\begin{abstract}
This paper presents two Tabu Search type algorithms for solving the multiprocessor scheduling problem. This problem consists in finding a schedule for a general task graph to be executed on a multiprocessor system so that the schedule length can be minimized. The multiprocessor scheduling problem is known to be NP-hard, and to obtain optimal and suboptimal solutions, several heuristic based algorithms have been developed in $[1,2,4,6]$. Our approaches are validated on 13 randomly generated instances. The numerical results show that our algorithms produce solutions closer to optimality and/or of better quality than the methods presented in [1].
\end{abstract}

\section{Introduction}

The problem of multiprocessor scheduling consists in finding a schedule for a general task graph (for example, figure 1) to be executed on a multiprocessor system so that the schedule length can be minimized. Each vertex is a task and edges are precedence relations between pairs of tasks. The sense of the arrow shows the direction of the precedence.

This scheduling problem is known to be NP-hard, and several approaches based on heuristic search have been developed to obtain optimal or (more often) suboptimal solutions. Effecient methods based on genetic algorithms, descent of gradient and Tabu Search [1, 2, 4, 5, 6] have been developed to solve the processor scheduling problem. [1] contains, to the best of our knowledge, the most efficient heuristics for the multiprocessor scheduling problem. This papers presents two Tabu Search type algorithms for solving more efficiently this problem than in [1].

\footnotetext{
* Nancy-Université, INRIA and LORIA, 54506 Vandoeuvre-les-Nancy, France. Email: buffet@loria.fr

† Nancy-Université, INRIA and LORIA, 54506 Vandoeuvre-les-Nancy, France. Email: cucu@loria.fr

†LMIA, Université de Haute-Alsace, 4 rue des Frères Lumière, 68093, Mulhouse, France. Email: lhassane.idoumghar@uha.fr

$\S$ Nancy-Université, Université Henri Poincaré, IECN and LORIA, 54506 Vandoeuvre-les-Nancy, France. Email: schott@loria.fr
}

\author{
Lhassane IDOUMGHAR ${ }^{\ddagger}$ René SCHOTT ${ }^{\S}$
}

The organization is as follows: Section 2 describes the model for multiprocessor schedulings. Section 3 recalls some hints on Tabu Search algoritms. In Section 4 we explain our contribution. Section 5 presents our experimental results and performance comparisons with the approach developped in [1]. Concluding remarks and further research aspects are contained in the last section.

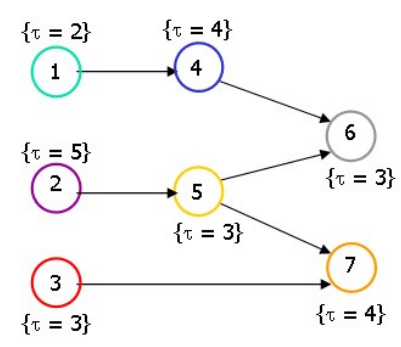

Figure 1. Graph of precedences.

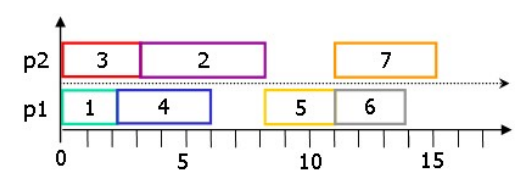

Figure 2. Example of scheduling.

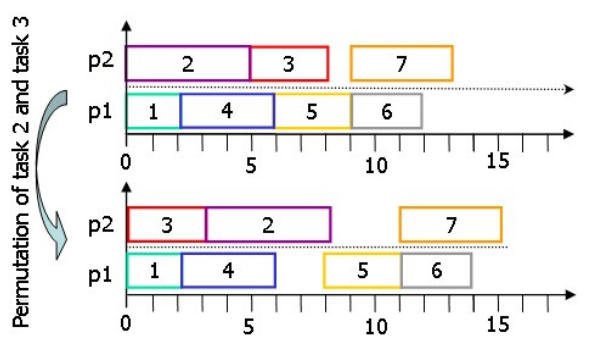

Figure 3. Permutation of two adjacent tasks. 


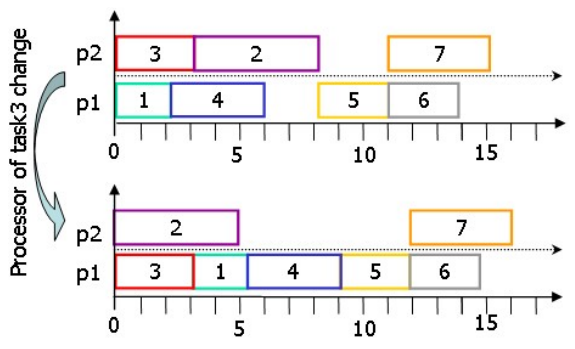

\section{Figure 4. Change of the processor attributed to task 3.}

\section{Model Description}

Consider a set of partially ordered computational tasks represented by a directed acyclic graph ${ }^{1}$ consisting of a finite nonempty set of vertices $V$ and a set of finite directed edges $E$ connecting the vertices.

The collection of vertices, $V=\left\{T_{1}, T_{2}, \ldots, T_{m}\right\}$ represents the set of computational tasks to be executed. Let $e_{i j}$ denote a directed edge from vertex $T_{i}$ to $T_{j}$. The set of directed edges, $E=\left\{e_{i j}\right\}$ implies that a partial ordering (or precedence relation), $\rightarrow$, exists between the tasks. $T_{i} \rightarrow T_{j}$ means that task $T_{i}$ must be completed before $T_{j}$ can be initiated.

The problem of optimaly scheduling a task graph onto a multiprocessor system with $p$ processors consists to assign the computational tasks to the processors, so that the precedence relations are maintained and all tasks are completed in shortest (possible) time. If task $T_{i}$ is an ancestor of task $T_{j}$ (i.e. if $T_{i}$ must be executed before $T_{j}$ ), then we say that $\operatorname{heigh}\left(T_{i}\right)<\operatorname{heigh}\left(T_{j}\right)$ where $\operatorname{heigh}\left(T_{i}\right)=0$ if the set $\operatorname{PREC}\left(T_{i}\right)$ of predecessors of $T_{i}$ is empty and $\operatorname{heigh}\left(T_{i}\right)=1+\max _{T_{j} \in P R E C\left(T_{j}\right)}\left(T_{j}\right)$ otherwise. This height function conveys the precedence relations between tasks. For example, in figure 1 we can see that:

- heigh $\left(T_{1}\right)=\operatorname{heigh}\left(T_{2}\right)=\operatorname{heigh}\left(T_{3}\right)=0$

- heigh $\left(T_{4}\right)=\operatorname{heigh}\left(T_{5}\right)=1$

- heigh $\left(T_{6}\right)=\operatorname{heigh}\left(T_{7}\right)=2$

\section{Tabu Search Algorithm}

The basic idea of the Tabu Search metaheuristic [3] is to explore the search space of all feasible solutions by a sequence of moves. A move from one solution to another is generally the best available. However, in order to prevent oscillation and to provide a mechanism for escaping from

\footnotetext{
${ }^{1}$ We use the graph model proposed in [4]
}

locally optimal but not globally optimal solutions, some moves, at one particular iteration, are classified as forbidden or tabu. Moves are regarded as tabu by consideration of short-term and long-term history of the sequence of moves. A very simple use of this idea might be to classify a move as tabu if the reverse move has been made recently or frequently. There is also an aspiration criterion, which overrides the tabu moves in particular circumstances. These circumstances might include the cases where, by forgetting that a move is tabu, a solution which is the best so far is obtained.

Suppose it is required to minimize some cost function $F$ on the search space $S$. For combinatorially hard problems it may only be possible to obtain sub-optimal solutions, in which $F$ is close to its minimum value. Sub-optimal problems may be obtained when a certain threshold for an acceptable solution has been achieved or when a certain number of iterations have been completed.

A characterisation of the search space $S$ for which Tabu Search can be applied is when there exists a set of $k$ moves $D=\left\{d_{1}, \ldots, d_{k}\right\}$ such that the application of the moves to a feasible solution $s \in S$ leads to k (usually distinct) solutions $D(s)=\left\{d_{1}(s), \ldots, d_{k}(s)\right\}$. The subset $N_{\text {set }}(s) \subseteq$ $D(s)$ of feasible solutions is known as the neighbourhood of $s$.

The method starts with a (possibly random) solution $s_{0} \in S$ and determines a sequence of solutions $s_{0}, s_{1}, \ldots, s_{n} \in S$. At each iteration, $s_{j+1}(0 \leq j<n)$ is selected from the neighbourhood $N_{\text {set }}\left(s_{j}\right)$. The selection process is first to determine the tabu set $T_{\text {set }}\left(s_{j}\right) \subseteq N_{\text {set }}\left(s_{j}\right)$ and the aspiration set $A_{\text {set }}\left(s_{j}\right) \subseteq N_{\text {set }}\left(s_{j}\right)$. Then $s_{j+1}$ is the neighbour of $s_{j}$ which is either an aspirant or not tabu and for which $F\left(s_{j+1}\right)$ is minimal; that is, $F\left(s_{j+1}\right) \leq$ $F\left(s_{i}\right)$ for all $s_{i} \in\left(N_{\text {set }}\left(s_{j}\right)-T_{\text {set }}\left(s_{j}\right)\right) \cup A_{\text {set }}\left(s_{j}\right)$.

In the next section we will present different elements used by our algorithm based on a refined modeling of the input data and on a Tabu Search.

\section{Our approach}

\subsubsection{Principle of our algorithm}

The principle of our Tabu Search algorithm is described in Algorithm 1, where:

- Initial solution: For the multiprocessor scheduling problem, a legal schedule is one that satisfies the following conditions:

1. The precedence relations among the tasks are satisfied.

2. Every task is present and appears only once in the schedule (completeness and uniqueness). 
The solution representation used in this paper is based on the schedule of the tasks on each individual processor [4]. This representation eliminates the need to consider the precedence relations between the tasks scheduled to different processors. The precedence relations within the processor, however, must still be maintained.

The representation of a schedule for Tabu Search Algorithms must accommodate the precedence relations between the computational tasks. This is resolved by representing the schedule as several lists of computational tasks. Each list corresponds to the computational tasks executed on a processor, and the order of the tasks in the list indicates the order of execution. Figure 2 illustrates the list representation of the schedule in Figure 1. This ordering allows us to maintain the precedence relations for the tasks executed on a processor (intraprocessor precedence relation) and ignores the precedence relations between tasks executed on different processors (interprocessor precedence relation). This is due to the fact that the interprocessor precedence relations do not come into play until we actually calculate the finishing time of the schedule. Each list can be further viewed as a specific permutation of the tasks in the list (allowing the last task to map to the first task). Figures 3 and 4 illustrate the permutation representation of the schedule in Figure 2. Thus, a schedule for $n$ tasks and $p$ processors is a permutation of $n$ numbers with $p$ cycles. The permutation representation of schedules is useful when we actually implement the function that generates the neighbouring solutions.

Note that not every permutation of $n$ numbers with $p$ cycles corresponds to a legal schedule because of the precedence relations. This representation of schedules falls into the following category: The solution space is not in one-to-one correspondence with the search space. We must bear this in mind when we design the neighbouring solutions.

- Variable best_solution keeps the best solution found,

- current_iteration counts the iterations of the main loop and Notimprove remembers the last iteration in which the algorithm improved the best solution.

- Evaluate or objective function : For the multiprocessor scheduling problem, we can consider factors such as throughput, finishing time, and processor utilization for the fitness function.

The objective function used for our TS approaches (also defined in [4]) is based on the finishing time of the schedule. The finishing time (FT) of a current schedule, $S_{c}$, is defined as follows:

$$
F T\left(S_{c}\right)=\max _{P_{j}} f t p\left(P_{j}\right)
$$

where $f t p\left(P_{j}\right)$ is the finishing time for the last task in processor $P_{j}$.

Thus, the optimal schedule founded by the Tabu Search algorithms must have the smallest finishing time value than the other schedules.

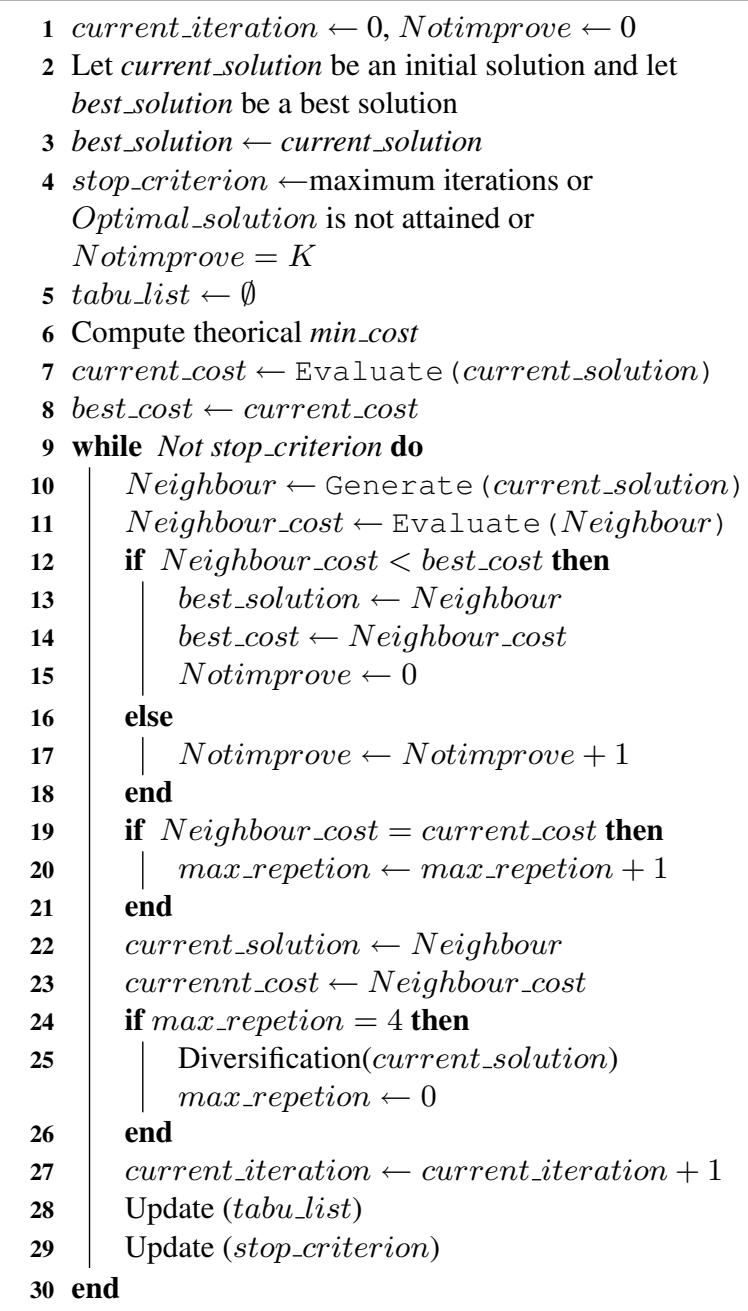

Algorithm 1: Our Tabu Search approach.

- Intensification: The role of the intensification is to drive the search towards interesting regions, by taking into account characteristics found in high quality solutions, in the selection of future solutions to visit. For each processor, our Tabu search algorithm checks if the possible permutation of the tasks would improve the quality of the current solution.

- Diversification: Tabu Search is based on Local Search principle. One of its main problems is that it spends most, if not all, of its time in a restricted region of the search space. 
In order to avoid that a large region of the search space remains completely unexplored, it is important to diversify the search.

In our approach, the diversification technique used tryes, randomly, either to penalize the current solution or to perform a random restart.

- The tabu_list is updated in line 28.

- stop_criterion: this criterion indicates when the search process will be stopped. The update procedure verifies the following conditions:

- maximum number of iterations is not attained

- or Optimal_solution is not found (best_cost $\neq$ min_cost)

- or Notimprove $<(K=60)$

Our first implementation (called TS_version1) of Tabu Search Algorithm consists in adding solutions to the tabu list (with size $=7$.)

The second implementation (called TS_version2) forbids some characteristics of the solutions. The neighbour of each solution is defined by two types of permutation (see Figures 3 and 4). To each of them we are going to associate a specific characteristic:

1. for each processor attributed to task $i$, there exist $i-1$ possible permutations between adjacent tasks, we identify them in a natural way by their position. When our Tabu Search algorithm escapes from a local minimum thanks to that modification, the corresponding permutation becomes tabu.

2. when our Tabu Search Algorithm escapes from a local minimum by changing the processor attributed to a task (for example, Figure 4), the previous attribution becomes tabu.

\section{Experimental results}

The Tabu Search algorithms have been implemented on a $P C$ centrino, with a $1.8 \mathrm{Ghz}$ processor and 512 Mbytes of memory running under Mandriva Linux 2007.

In order to compare the different scheduling approaches, we have generated randomly systems of 50 tasks. A total of 10 runs for each algorithm were conducted and the number of processors used to solve each problem is fixed to 10 .

The best results obtained by all algorithms are given in Figures 5, 6 and 7.

Analysing theses figures, we can see:

- In Figure 5, TS_version 1 produces almost every time the optimal solution for almost all instances and improves DGV ([1]).

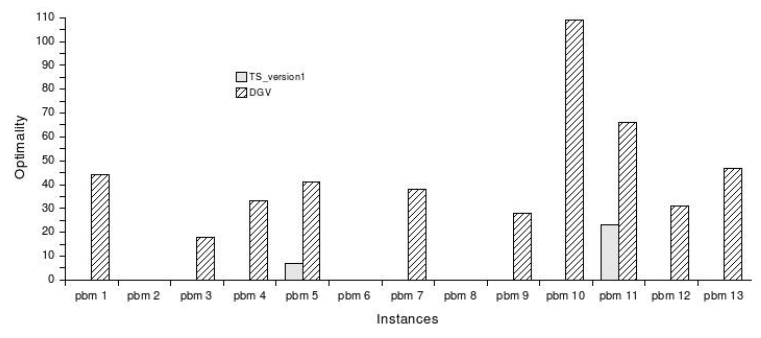

Figure 5. Measure of the distance to the optimal solution.

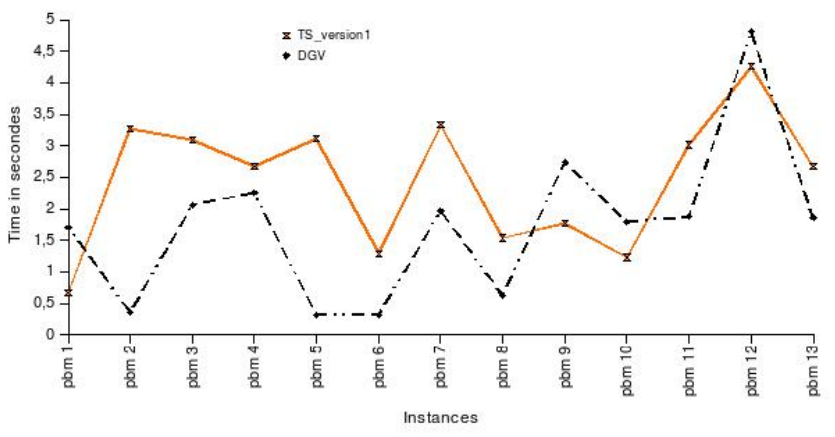

Figure 6. Comparison of computation times.

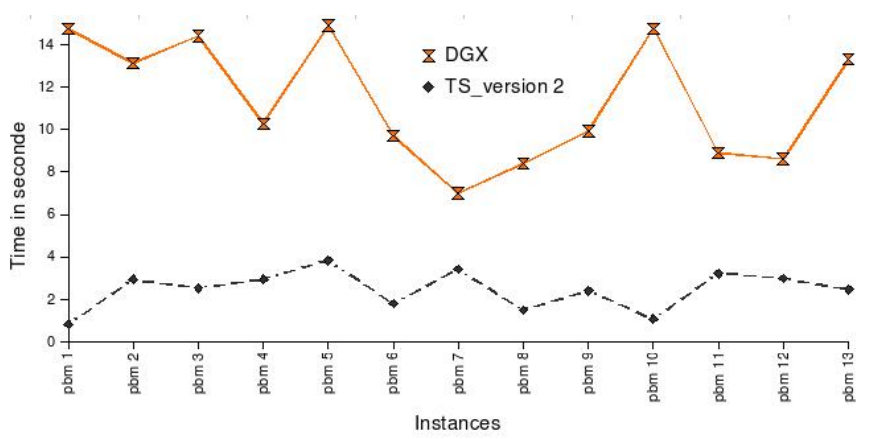

Figure 7. Comparison of computation times.

- In Figure 6, For $77 \%$ of the examples considered, TS_version 1 provides rapidly a better solution than $D G V$ ([1]). For the $23 \%$ remaining cases, $T S \_v e r$ sion 1 takes a little bit more time than $D G V$ but produces solutions of better quality than $D G V$.

- In Figure 7, TS_version2 provides more quickly a better solution than $D G X$ ([1]). The improvements of computation times are do to the stopping criterion as well as to programming enhancements (for example, avoiding calculation of the cost at each iteration). DGX ([1]) and TS_version2 produce both the same good solution but TS_version2 needs less time. 


\section{Concluding remarks}

Our Tabu Search type algorithms improve drastically the performances of these presented in [1]. We are currently implementing our algorithm on clusters of PC's. Further research aspects include the design of a probabilist Tabu Search Algorithm for the multiprocessor problem as well as the use of Markov Decision Processes (MDP's) and Particle Swarm Optimization (PSO). Designing hybrid algorithms for solving this problem is a real challenge.

\section{References}

[1] B. Chauvière, D. Geniet and R. Schott, Contributions to the Multiprocessor Scheduling Problem, Proceedings of 3rd IASTED International Conference on Computational Intelligence, Banff, Canada, July 2-4, 2007, 5560.

[2] R.C. Corrêa, A. Frerreira and P. Rebreyend, Scheduling Multiprocessor Tasks with Genetic Algorithms, IEEE Transaction on Parallel and Distributed Systems, 10, 8, 1999, 825-837.

[3] F. Glover and E. Taillard, A User's Guide to Tabu Search, Annals of Operations Research, 41, 1993, 3-28.

[4] E.S. Hou, N. Ansari and H. Ren, A Genetic Algorithm for Multiprocessor Scheduling,

IEEE Transaction on Parallel and Distributed Systems, 5, 2, 1994, 113-120.

[5] Y. Monnier, J-P. Beauvais and A.M. Déplanche, A Genetic Algorithm for Scheduling Tasks in a Real-Time Distributed System, Proceedings of the 24th EUROMICRO Conference (EUROMICRO'98), IEEE Pub.

[6] A.S. Wu, H. Yu, S. Jin, K-C. Lin and G. Sciavone, An Incrimental Genetic Algorithm Approach to Multiprocessor Scheduling, IEEE Transaction on Parallel and Distributed Systems, 15, 9, 2004, 824-834. 